\title{
When Zebras Run with Horses: The Diagnostic Dilemma of Acute Aortic Dissection Complicated by Myocardial Infarction
}

\author{
BRAHMAJEE K. NALLAMOTHU, M.D., MPH, and KIM A. EAGLE, M.D. \\ From the Department of Internal Medicine, University of Michigan Medical School, Ann Arbor, Michigan
}

\begin{abstract}
In this issue of the Journal, Pinney and Wasserman ${ }^{l}$ describe the case of a patient who survives acute aortic dissection complicated by occlusion of the left main coronary ostium and anterior myocardial infarction (MI), due in part to the fortuitous presence of an anomalous left circumflex artery. The case illustrates the challenges of diagnosing aortic dissection, even in the current era of accurate and rapid aortic imaging, in individuals with concurrent acute MI. These comments are focused in this comment on two key aspects of the case: (1) How commonly do acute aortic dissections lead to myocardial ischemia and MI? and (2) When should clinicians consider ruling out acute aortic dissection in the setting of acute MI? (J Interven Cardiol 2002;15:297-300)
\end{abstract}

The earliest reports on aortic dissection suggested that concurrent MI was rare, occurring in $<2 \%$ of all cases. $^{2-4}$ Using the International Registry of Acute Aortic Dissection (IRAD), Hagan et al. ${ }^{5}$ recently found acute $\mathrm{MI}$ in nearly 5\% of patients presenting with type A aortic dissection. The IRAD registry was limited (as were earlier studies) primarily by a narrow definition of acute MI, which included the development of new $Q$ waves or ST segment elevations on electrocardiogram but not routine testing for elevations in serum biomarkers like cardiac-specific troponins or creatine kinase, isoenzyme ${ }^{-2}$ (CKMB). Thus, non-ST segment elevation MI was inconsistently detected in these studies under the above criteria. Further complicating the issue is the fact that nonspecific electrocardiographic changes, contrary to earlier teaching, are common in both diagnoses. In fact, only $31 \%$ of patients with aortic dissection in the IRAD registry had completely normal electrocardiograms. ${ }^{5}$ While the great majority of abnormal electrocardiograms in that study demonstrated nonspecific

Address for reprints: Kim A. Eagle, M.D., Div. of Cardiology, Dept. of Internal Medicine, University of Michigan Medical Center, 3910 Taubman Ctr., Ann Arbor, MI 48109-0366. Fax: (734) 936-8944; email: keagle@umich.edu
ST or T-wave changes (including left ventricular hypertrophy with strain), $15 \%$ were consistent with myocardial ischemia. ${ }^{5}$

Patients with acute aortic dissection are at risk for ST segment elevation and non-ST segment elevation MI. In the first situation, differentiating between an acute MI due to coronary ostia compression from an aortic dissection flap and a more typical MI from plaque rupture and thrombosis is critical, since inappropriately administering or delaying therapies for either disease is potentially life threatening. Recognizing that the overall incidence of acute MI is nearly 800 times higher than the estimated incidence of acute aortic dissection in the United States, ${ }^{5}$ and that acute ST elevation MI is a rare complication of aortic dissection, several investigators, including Pinney and Wasserman, correctly point out that delaying time dependent treatments like thrombolysis to rule out aortic dissection in all patients with acute ST elevation MI would be a mistake. ${ }^{1,6}$ Further evaluation in patients with symptoms suggestive of aortic dissection, however, is necessary.

However, in patients with non-ST elevation MI, it makes sense to be more cautious and to at least consider aortic imaging, as dissection is statistically more likely in the setting of nonspecific electrocardiographic changes. Furthermore, delaying therapy (e.g., 
anticoagulant and antiplatelet agents) in these patients for a short period of time is not likely to be critical. Again, ruling out aortic dissection in all patients with non-ST elevation MI would be a mistake.

So when should clinicians consider the possibility of aortic dissection in the patient with chest pain and an abnormal electrocardiogram? Frankly, this question cannot be answered precisely since this decision relies more on the art of clinical diagnosis rather than the science of probabilistic decision making. Nevertheless, the clinical history, physical examination, and simple laboratory tests should direct the initial evaluation. Von Kodolitsch et al., ${ }^{7}$ for instance, found three important clinical variables that identified $96 \%$ of the aortic dissections in their case series of 250 patients with suspicious symptoms: (1) the presence of "aortic pain," described as the abrupt or "instantaneous" onset of chest or back pain with or without a tearing or ripping character; (2) pulse or blood pressure differentials; and (3) mediastinal widening, aortic widening, or both on chest radiography. Of these three variables, abrupt symptom onset seems the most sensitive clinical finding, being described in over $80 \%$ of patients with aortic dissection. ${ }^{5.7}$ Others have suggested that a history of hypertension and migratory pain or the presence of an aortic regurgitation murmur also might be helpful in identifying dissection early. ${ }^{3-5}$ Finally, risk factors such as Marfan syndrome or prior cardiac surgery should always alert clinicians to the possibility of dissection as well.

Curiously, the patient in the case described by Pinney and Wasserman ${ }^{1}$ had a "sudden" onset of chest pain that unfortunately was not further characterized. Chest radiography and pulse or blood pressure differentials were also not documented, and no aortic regurgitation murmur was appreciated at the time of initial examination. Whether these additional findings would have helped identify aortic dissection any earlier in this patient will never be known; however, clinicians should at the very least keep these clinical factors in mind, particularly before starting thrombolytic or anticoagulant therapy.

If aortic dissection is considered in a patient, transesophageal echocardiography (TEE), contrast enhanced $\mathrm{x}$-ray computed tomography (CT), and magnetic resonance imaging remain the initial tests of choice for establishing a definitive diagnosis. In the setting of a type A dissection, the type most important to rule out in the setting of an acute ST elevation MI, transthoracic echocardiography (TTE) has a reported sensitivity of $78-100 \%^{8}$ and could be performed rapidly at the bedside. A TTE likely would have raised suspicion of an aortic dissection in the patient described above given his severe aortic insufficiency. It also might have identified his proximal dissection flap. In the future, testing for serum biomarkers with rapid smooth muscle myosin heavy-chain protein assays might be another option for excluding aortic dissection in patients with suspicious symptoms in the emergency department. ${ }^{9}$

Up to $7 \%$ of emergency department visits in the United States are for the evaluation of chest pain. ${ }^{10}$ Two potentially fatal conditions associated with chest pain are acute MI and aortic dissection. Distinguishing between these two conditions is difficult enough when each exist in isolation, but becomes even more challenging when the two are present simultaneously in a patient. Bearing in mind that statistically an acute MI is much more likely to be encountered than aortic dissection, the authors agree with Pinney and Wasser$\operatorname{man}^{1}$ that general policies which screen for "zebras" at the expense of delaying time dependent treatments for "horses" would be a mistake. Instead, clinicians need to focus on selected cases (i.e., patients with an abrupt onset of chest pain), particularly when accompanied by known risk factors for aortic dissection (e.g., hypertension, Marfan syndrome) and abnormal physical examination (e.g., pulse differentials, aortic regurgitation murmur) or chest radiography findings.

Formal studies that aid in distinguishing between these two diseases are clearly needed, but understandably difficult to carry out given the rare nature of aortic dissection. Until better information is available the lesson gleaned from the described case is worthwhile: Maintain suspicion for aortic dissection, even when the electrocardiogram clearly demonstrates myocardial ischemia or MI, and carefully consider rapid aortic imaging in select cases before initiating thrombolytic or anticoagulant therapy.

Acknowledgments:The authors thank Sanjay Saint, M.D., MPH and Rajendra H. Mehta, M.D., MSc for their thoughtful suggestions.

\section{References}

1. Pinney SP, Wasserman HS. Anterior myocardial infarction, acute aortic dissection and anomalous coronary artery. J Interventional Cardiol 2002;15:293-296.

2. Hirst AE Jr, Johns VJ Jr, Kime SW Jr. Dissecting aneurysm of the aorta: A review of 505 cases. Medicine 1958;37:217-219. 
3. Slater EE, DeSanctis RW. The clinical recognition of dissecting aortic aneurysm. Am J Med 1976;60:625-633.

4. Spittell PC, Spittell JA, Joyce JW, et al. Clinical features and differential diagnosis of aortic dissection: Experience with 236 cases (1980 through 1990). Mayo Clin Proc $1993 ; 68: 642-651$.

5. Hagan PG, Nienaber CA, Isselbacher EM, et al. The International Registry of acute aortic dissection (IRAD): New insights into an old disease. JAMA 2000;283:897-903.

6. Kamp TJ, Goldschmidt-Clermont PJ, Brinker JA, et al. Myocardial infarction, aortic dissection, and thrombolytic therapy. Am Heart J 1994;128:1234-1237.

7. von Kodolitsch Y, Schwartz AG, Nienaber CA. Clinical pre- diction of acute aortic dissection. Arch Intern Med 2000;160:2977-2982.

8. Cigarroa JE, Isselbacher EM, DeSanctis RW, et al. Diagnostic imaging in the evaluation of suspected aortic dissection: Old standards and new directions. N Engl J Med 1993;328;35-43.

9. Suzuki T, Katoh H, Tsuchio Y, et al. Diagnostic implications of elevated levels of smooth-muscle myosin heavy-chain protein in acute aortic dissection. Ann Intern Med 2000;133:537-541.

10. American College of Emergency Physicians: Clinical policy for the initial approach to adults presenting with a chief complaint of chest pain, with no history of trauma. Ann Emerg Med 1995;25:274-299. 